\title{
Development of organic-inorganic halide perovskites (OHPs) based memristors
}

Mohammed Al-hor, Rana Magdy, Zubair Ahmad, Noora Al-Thani

Faculty and Postdoc., Energy and Environment

\section{Abstract}

\section{Introduction/Objective}

MAPbl 3 has been the well known OHP in the field of thin film-based photovoltaic device (PVD). However, shape and size of crystals have profound impacts on electrical and optical properties. Hence lower dimension (zero-dimension, one dimension) OHP crystal structure provide particular set of advantages such as large surface to volume ratio, better charge separation and conductivity makes OHP very useful in optoelectronics application other then PVD. The morphological, and structural properties of the one-dimensional perovskite micro-rods have been examined using SEM and XRD characterization techniques. Memory resistors has been fabricated using micro-rods. Current - Voltage (IV) characteristics has been studied to examine charge conduction behavior of the device

\section{Hypothesis}
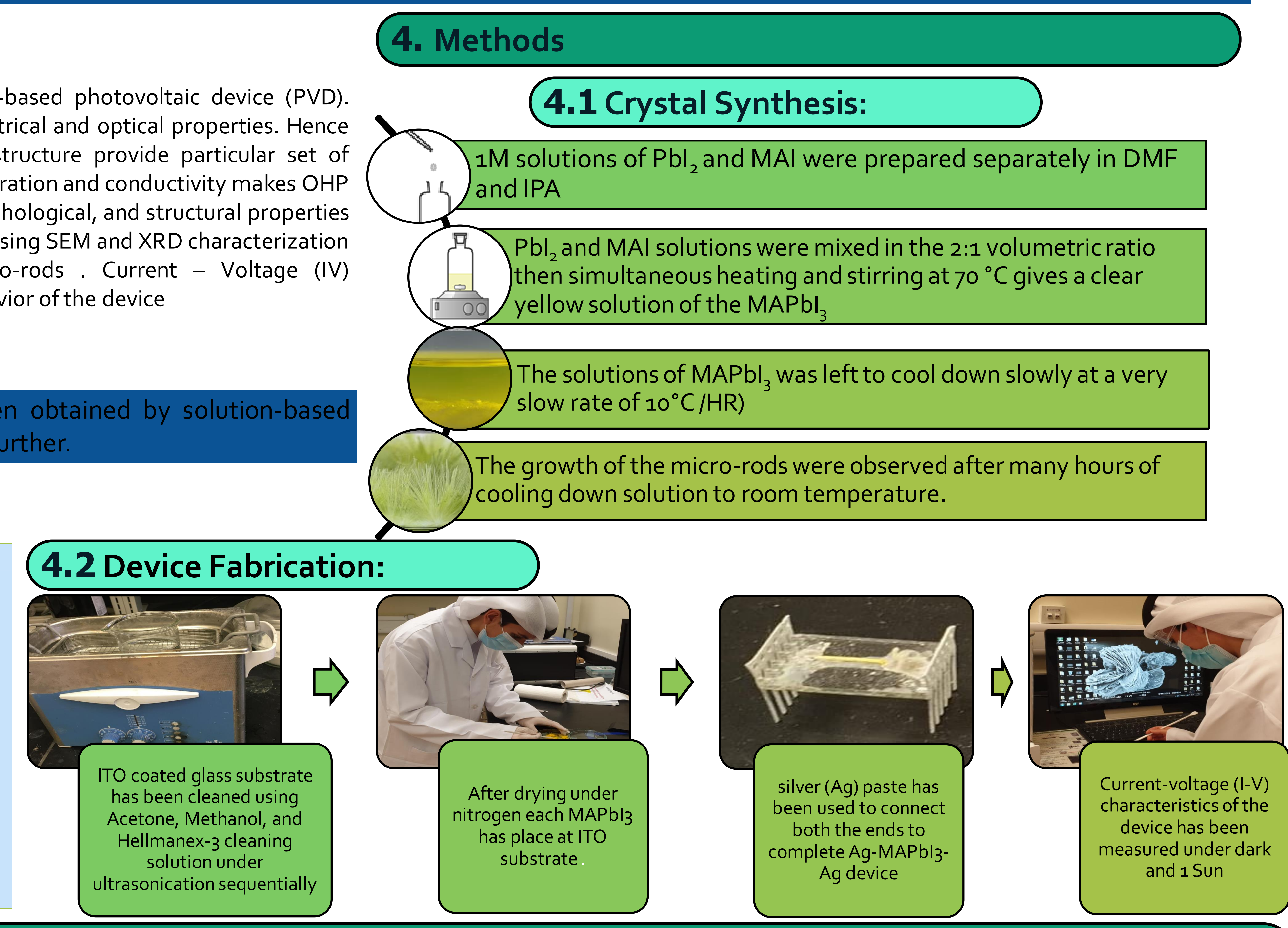

5. Results and Analysis

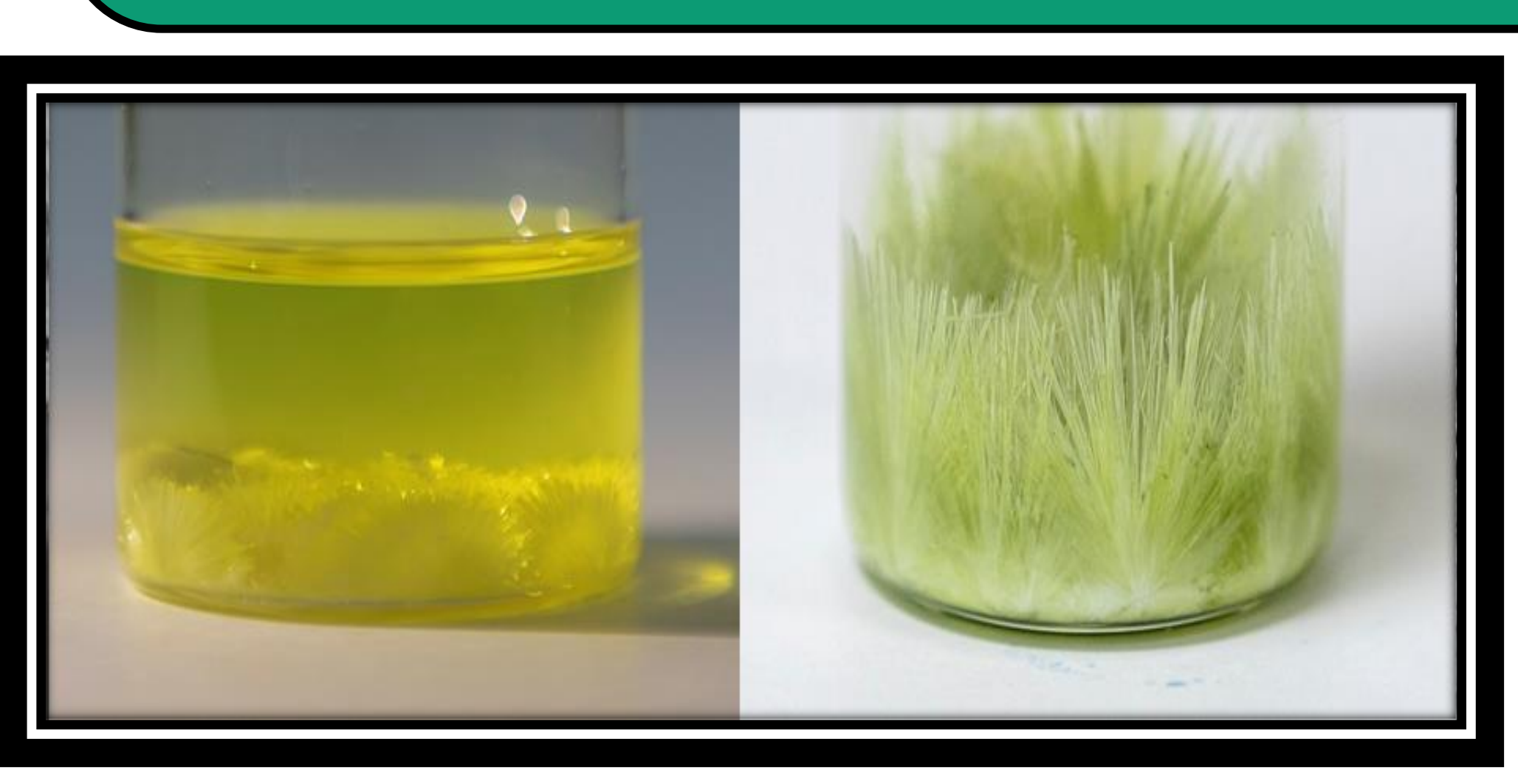
the DMF solution.

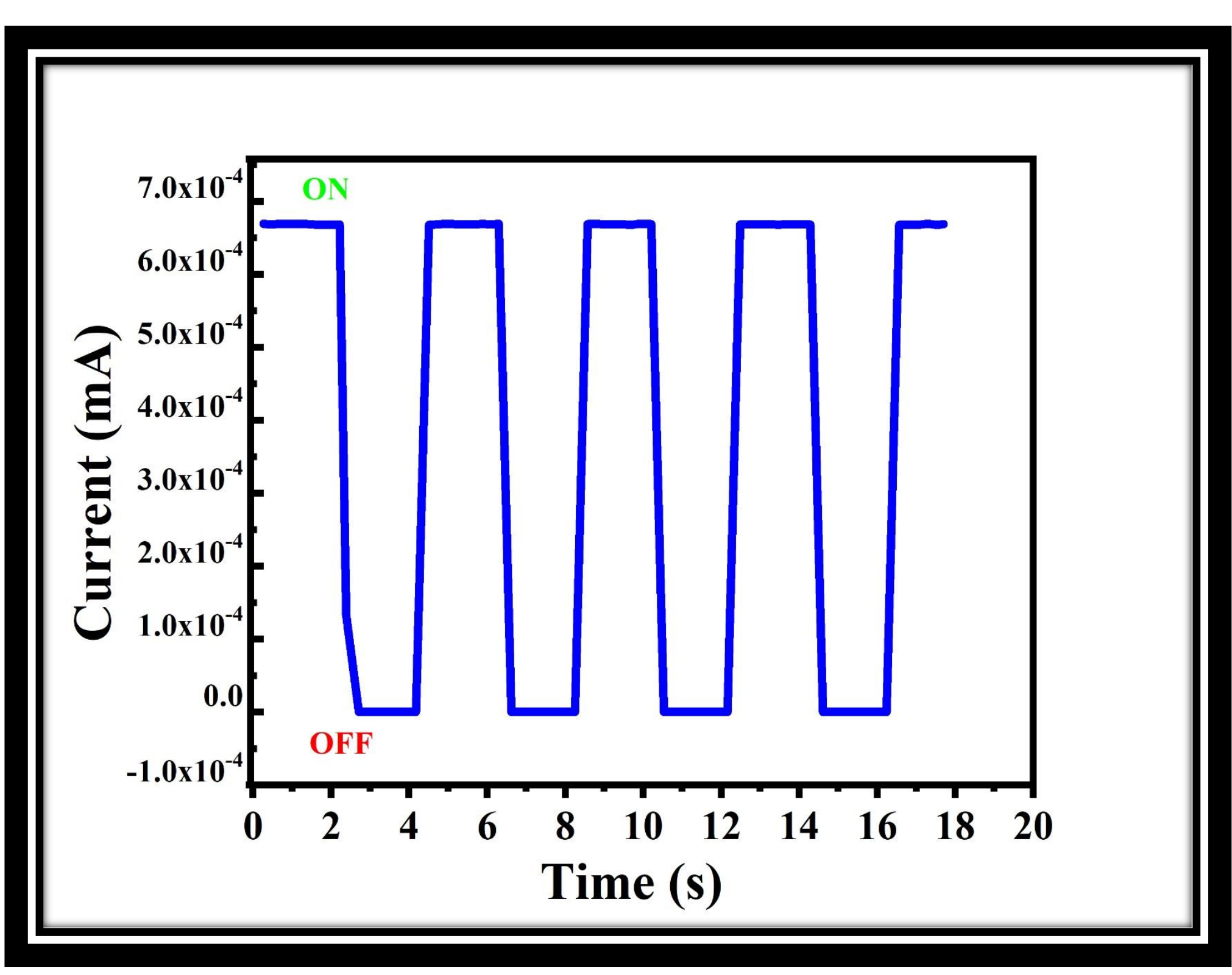

Figure 6: ON/OFF behaviour of $\mathrm{Ag} / \mathrm{MAPbl} / \mathrm{Ag}$ based memory resistor .

\section{Concluding remarks}

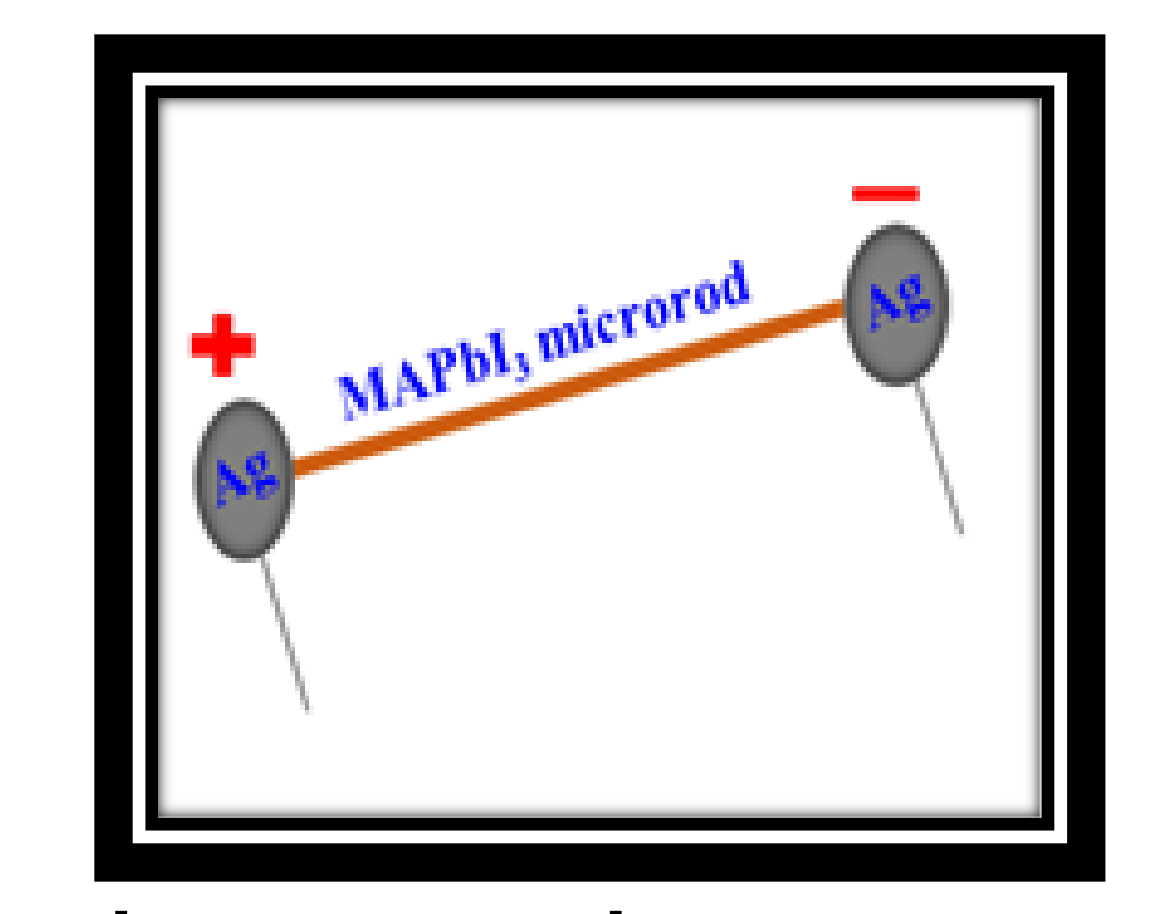

igure 5: Device Structure resistor.
Figure 1: MAPb/3 crystals grown in

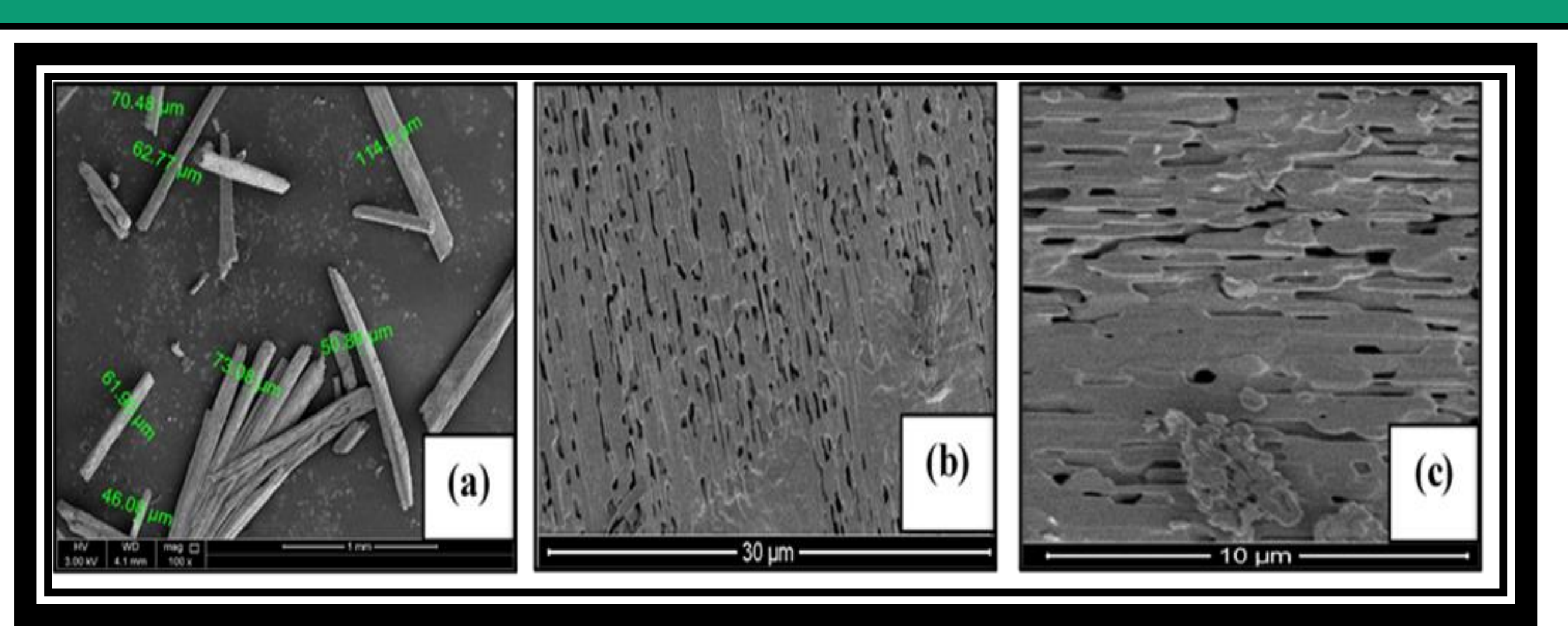

Figure 2: FESEM images of $\mathrm{MAPbl}_{3}$ micro-rods taken at different magnification. ( $a, b$ and $c$ ).

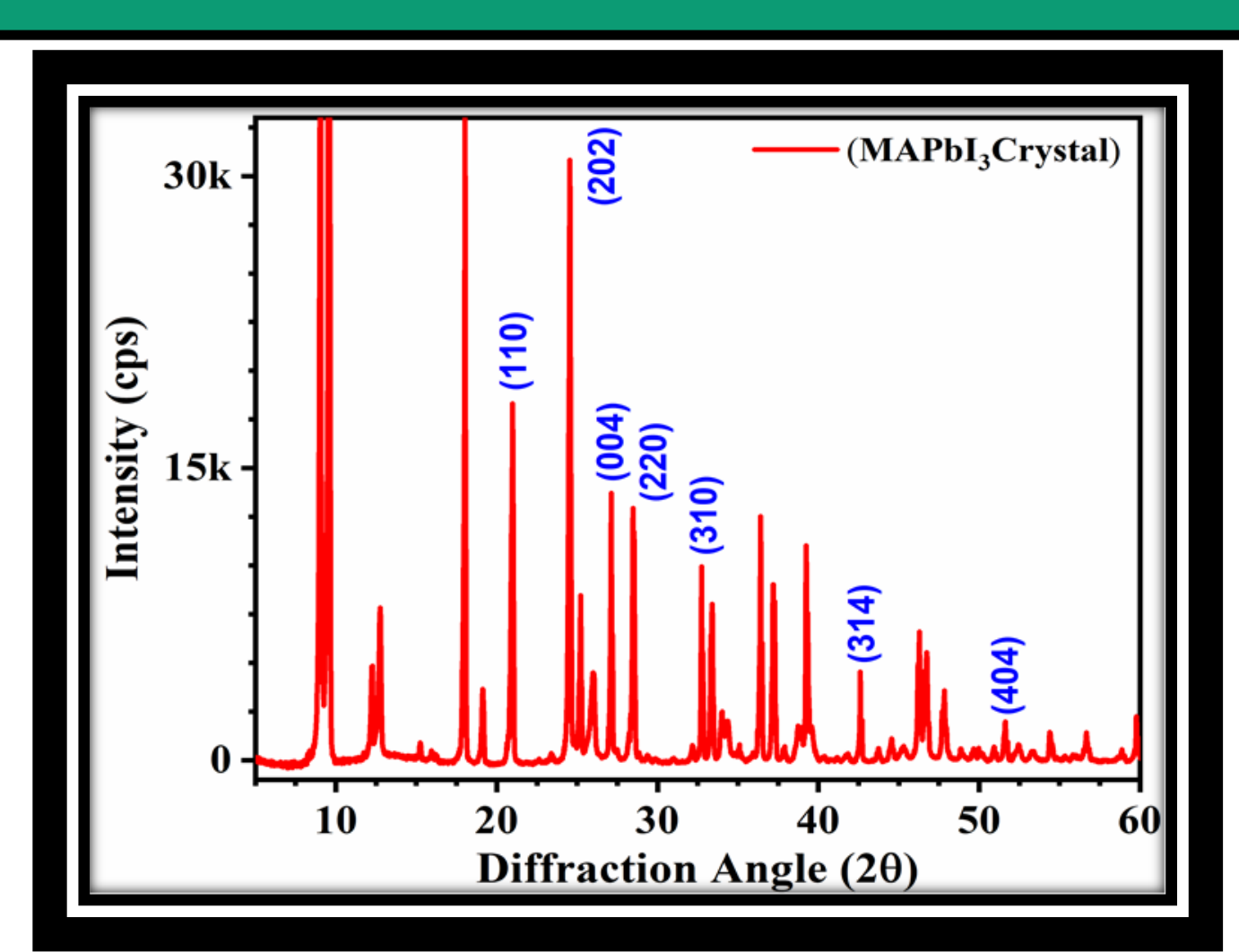

Figure $3: \mathrm{XRD}$ results of $\mathrm{MAPbl}_{3}$ micro rods.

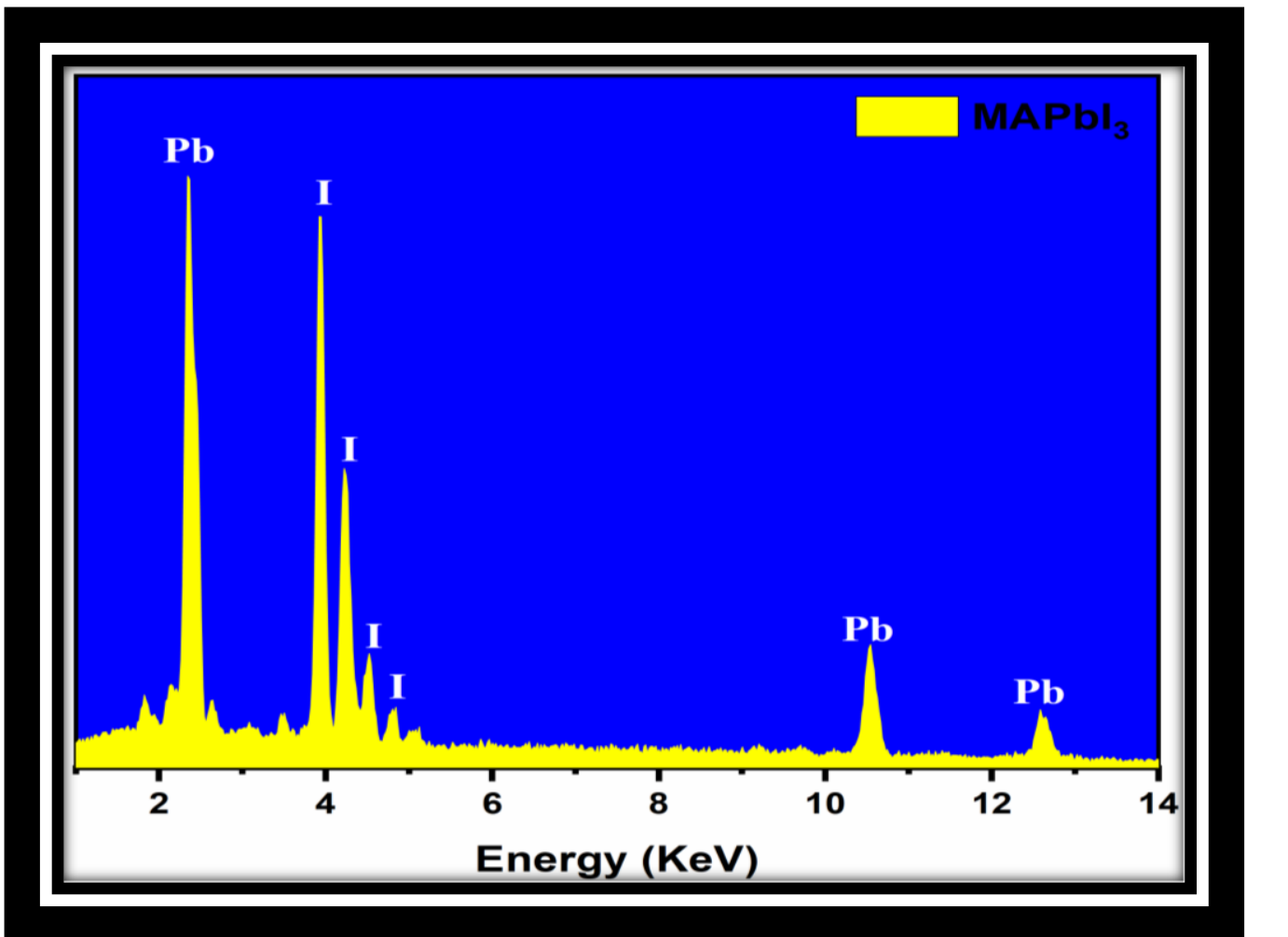

Figure 4: EDS spectra of the $\mathrm{MAPbl}_{3}$ micro-rods. of Ag/MAPbl3/Ag based

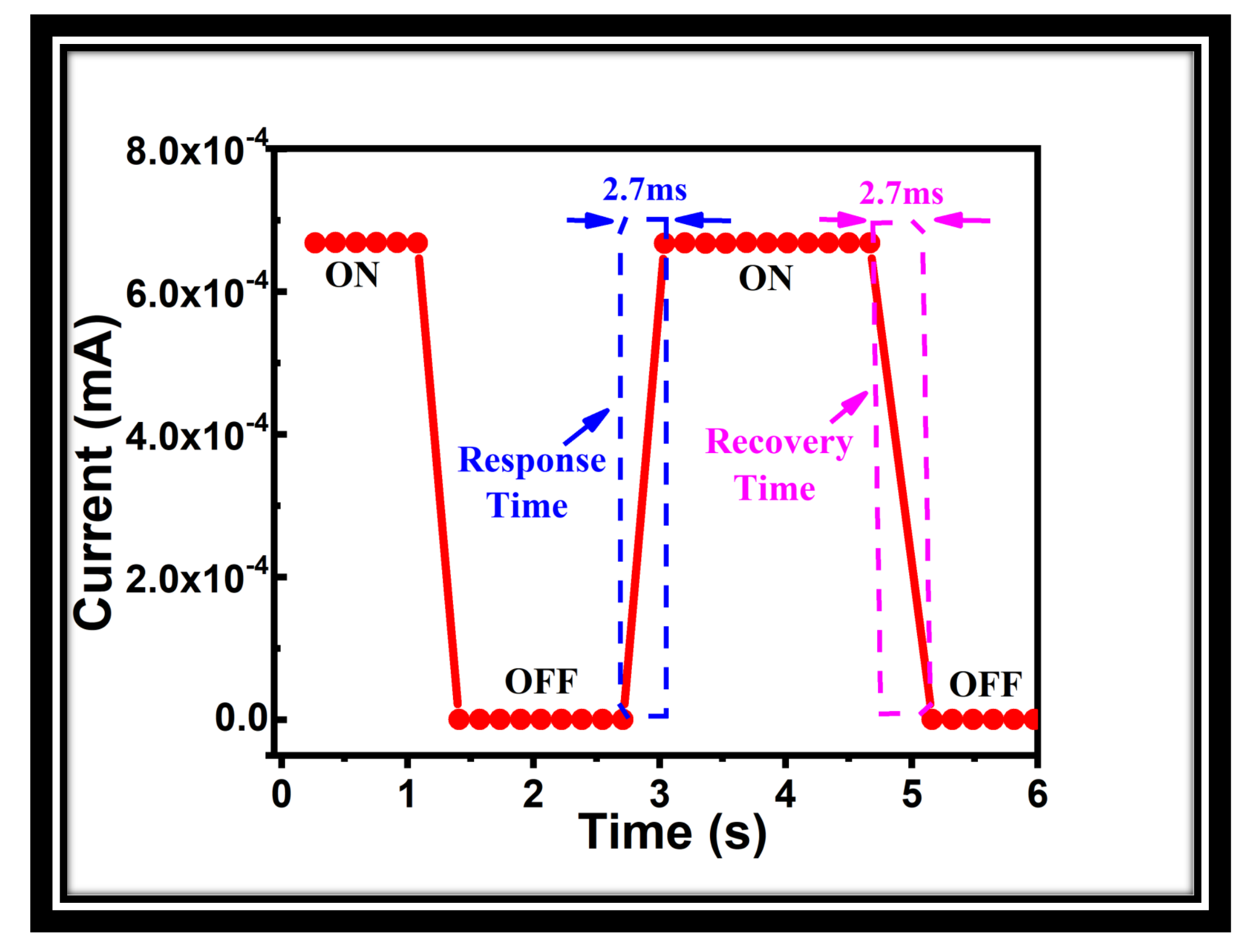

Figure 7: Response and recovery switching cycles of device.

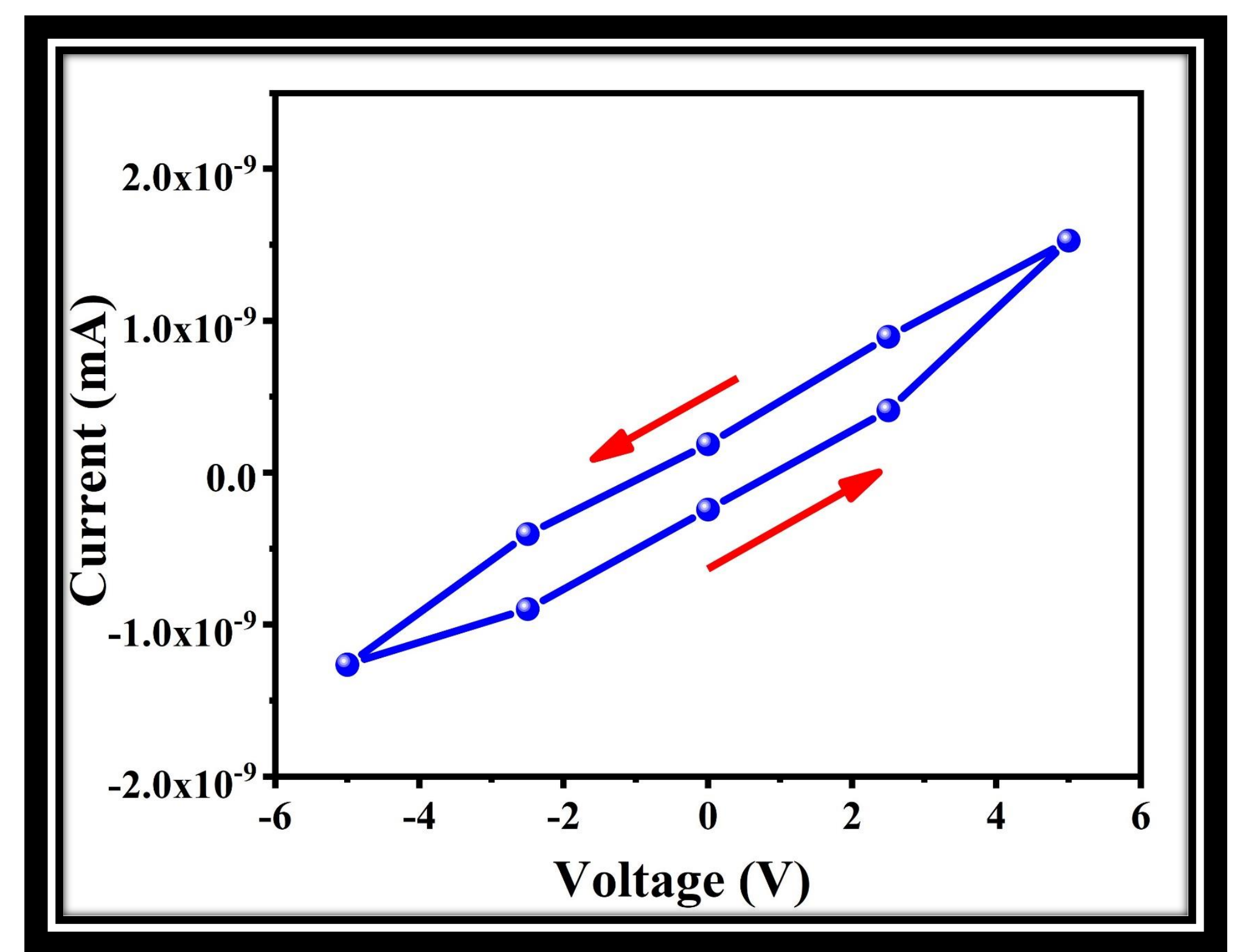

Figure 8: Hysteresis plot for Ag/MAPbl3/Ag based device.

\section{References}

[1] Kim H, Han J S, Kim S G, Kim S Y and Jang H W J J o M C C 2019 Halide perovskites for resistive random access memories 75226

2] Rangelow A S M IS M a IW 2019 Probe-induced resistive switching memory based on organic-inorganic lead halide perovskite materials Organic Electronics

[3] Zhao J, Li S, Tong W, Chen G, Xiao Y, Lei S and Cheng B 2018 Light-Induced Anomalous Resistive Switches Based on Individual Organic-Inorganic Halide Perovskite Micro-/Nanofibers Advanced Electronic Materials 41800206

[4] Kim D J, Tak Y J, Kim W G, Kim J K, Kim J H and Kim H J 2017 Resistive switching properties through iodine migrations of a hybrid perovskite insulating layer Advanced Materials Interfaces 41601035

[5] Liu Y, Li F, Chen Z, Guo T, Wu C and Kim T W 2016 Resistive switching memory based on organic/inorganic hybrid perovskite materials Vacuum 13010 